\title{
On the maximum values of the additive representation functions
}

\author{
Sándor Z. Kiss ${ }^{*}$ Csaba Sándor ${ }^{\dagger}$
}

\begin{abstract}
Let $A$ and $B$ be sets of nonnegative integers. For a positive integer $n$ let $R_{A}(n)$ denote the number of representations of $n$ as the sum of two terms from $A$. Let

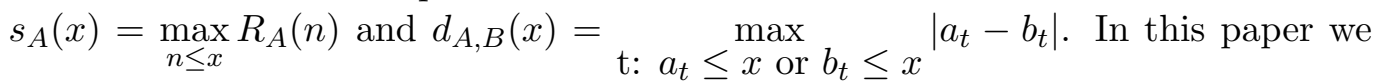
study the connection between $s_{A}(x), s_{B}(x)$ and $d_{A, B}(x)$. We improve a result of Haddad and Helou about the Erdős - Turán conjecture.
\end{abstract}

2000 AMS Mathematics subject classification number: 11B34. Key words and phrases: additive number theory, representation functions, Erdős - Turán conjecture.

\section{Introduction}

Let $\mathbb{N}$ denote the set of nonnegative integers. Let $A=\left\{a_{1}, a_{2}, \ldots\right\}, 0 \leq a_{1}<a_{2}<\ldots$, $B=\left\{b_{1}, b_{2}, \ldots\right\}, 0 \leq b_{1}<b_{2}<\ldots$ be infinite sequences of nonnegative integers. Let $R_{A}(n)$ denote the number of solutions of the equation

$$
a_{i}+a_{j}=n, \quad a_{i}, a_{j} \in A, \quad i \leq j,
$$

where $n \in \mathbb{N}$. Let $A(x)$ denote the number of elements of the sequence $A$ up to $x$. A (finite or infinite) set $A$ of positive integers is said to be a Sidon set if all the sums $a+b$ with $a, b \in A, a \leq b$ are distinct. Let $s_{A}(x)=\max _{n \leq x} R_{A}(n)$ and $s_{A}=\lim _{x \rightarrow \infty} s_{A}(x)$, $d_{A, B}(x)=\max _{\mathrm{t}: a_{t} \leq x \text { or } b_{t} \leq x}\left|a_{t}-b_{t}\right|, d_{A, B}=\lim _{x \rightarrow \infty} d_{A, B}(x)$. Throughout the paper we use the following usual notations. If $f$ and $g$ are real functions, then $f \ll g$ means that $f=O(g)$. If there exist positive constants $c_{1}$ and $c_{2}$ such that $c_{1} g \leq f \leq c_{2} g$ then we write $f=\Theta(g)$. We write $f \sim g$ if the function $f$ is asymptotically equal to $g$.

In 1941 Erdös and Turán [7] posed the following conjecture: if $R_{A}(n)$ is positive from a certain point on, then it cannot be bounded. Despite all the efforts this conjecture is still unsolved. In [8] and [9] Erdös and Fuchs formulated the following conjecture which is a little bit stronger then the original conjecture of Erdős and Turán.

*Institute of Mathematics, Budapest University of Technology and Economics, H-1529 B.O. Box, Hungary; Computer and Automation Research Institute of the Hungarian Academy of Sciences, Budapest H1111, Lágymányosi street 11; kisspest@cs.elte.hu; MTA-BME Lendület Future Internet Research Group; This author was supported by the OTKA Grant No. NK105645.

$\dagger$ Institute of Mathematics, Budapest University of Technology and Economics, H-1529 B.O. Box, Hungary, csandor@math.bme.hu. This author was supported by the OTKA Grant No. K109789. This paper was supported by the János Bolyai Research Scholarship of the Hungarian Academy of Sciences. 
Conjecture 1. For any $\mathcal{A}=\left\{a_{1}, a_{2}, \ldots\right\}$ set of nonnegative integers with $a_{n} \leq c n^{2}$ for all $n$ and $c>0$ real constant, we have $\limsup _{n \rightarrow \infty} R_{A}(n)=\infty$.

It is clear that this conjecture implies the original conjecture of Erdős and Turán. However, a lot of partial results has been made about the Erdös - Turán conjecture $[2,3,4,5$, $6,7,8,9,10,15,16,18,19,20,21,22,24,25,26,28]$, very little progress has been made about the generalized Erdős - Turán conjecture. In [10], [12] Grekos, Haddad, Helou and Pihko proved several statements that are equivalent to the generalized Erdős - Turán conjecture. In particular, they proved [12], [13] that the conjecture is true if $a_{n}=o\left(n^{2}\right)$. In [16] Haddad and Helou proved the following theorem:

Theorem 1 (Haddad, Helou, 2012). If $\left|a_{n}-n^{2}\right|=o(\sqrt{\log n})$, or in general $\left|a_{n}-q(n)\right|=$ $o(\sqrt{\log n})$, where $q(n)$ is a quadratic polynomial with rational coefficients, then $R_{A}(n)$ is unbounded.

In this paper we improve on their result by proving that

Theorem 2. For an arbitrary $\varepsilon>0$ if $A \subset \mathbb{N}, A=\left\{a_{1}, a_{2}, \ldots\right\}$ such that $\left|a_{n}-n^{2}\right|=$ $O\left(e^{(\log 2-\varepsilon) \frac{\log n}{\log \log n}}\right)$, then $R_{A}(n)$ cannot be bounded.

We conjecture that this result can be generalized.

Problem 1. Let $q(n)$ be a real quadratic polynomial with positive leading coefficient. Is it true that for $\left|a_{n}-q(n)\right|=O\left(e^{(\log 2-\varepsilon) \frac{\log n}{\log \log n}}\right)$ we have $s_{A}=\infty$ ?

The aim of this paper is to study the connections between the functions $s_{A}(x), d_{A, B}(x)$ and $s_{B}(x)$. We distinguish four cases according to $s_{A}$ and $d_{A, B}$ are finite or infinite. Theorem 3. deals with the case $s_{A}$ and $d_{A, B}$ are finite. We determine all the possible triplets $\left(s_{A}, s_{B}, d_{A, B}\right)$. Note that the first part of Theorem 3. is Corollary 3. in [12].

Theorem 3. 1. Let $A, B \subset \mathbb{N}$ such that $s_{A}<\infty, d_{A, B}<\infty$. Then we have

$$
\frac{s_{A}}{4 d_{A, B}+1} \leq s_{B} \leq\left(4 d_{A, B}+1\right) s_{A} .
$$

2. Let $a, b, d \in \mathbb{Z}^{+}$such that

$$
\frac{a}{4 d+1} \leq b \leq(4 d+1) a .
$$

Then there exist $A, B \subset \mathbb{N}$ such that $s_{A}=a, s_{B}=b, d_{A, B}=d$.

The following theorem deals with the case when the representation function $R_{A}(n)$ is unbounded, but the distance $d_{A, B}(n)$ is bounded.

Theorem 4. 1. Let $A, B \subset \mathbb{N}$ such that $s_{A}=+\infty, d_{A, B}<+\infty$. Then

$$
\frac{1}{4 d_{A, B}+1} \leq \liminf _{x \rightarrow \infty} \frac{s_{B}(x)}{s_{A}\left(x-2 d_{A, B}\right)} \leq \limsup _{x \rightarrow \infty} \frac{s_{B}(x)}{s_{A}\left(x+2 d_{A, B}\right)} \leq 4 d_{A, B}+1
$$


2. Let $d$ be a positve integer and $\alpha, \beta$ positive real numbers such that $\frac{1}{4 d+1} \leq \alpha \leq \beta \leq$ $4 d+1$. Then there exist $A, B \subset \mathbb{N}$ such that $s_{A}=+\infty, d_{A, B}=d$ and

$$
\liminf _{x \rightarrow \infty} \frac{s_{B}(x)}{s_{A}(x-2 d)}=\alpha, \quad \limsup _{x \rightarrow \infty} \frac{s_{B}(x)}{s_{A}(x+2 d)}=\beta
$$

The next theorem shows that for a finite $s_{A}$ and finite or infinite $s_{B}$ the rate of the unbounded distance $d_{A, B}(n)$ can be arbitrary small.

Theorem 5. Let $a \in \mathbb{Z}^{+}, b \in \mathbb{Z}^{+} \cup\{\infty\}$ and let $f: \mathbb{Z}^{+} \rightarrow \mathbb{N}$ be a monoton increasing function with $f(n) \rightarrow \infty$. Then there exist $A, B \subset \mathbb{N}$ such that $s_{A}=a, s_{B}=b$ and $\left|a_{n}-b_{n}\right| \leq f(n)$. The last inequality implies, that $d_{A, B}(n-1) \leq f(n)$.

The right hand side of inequality (1) in Lemma 1. implies that for a function $f(n)$ and for Sidon set $A$ and set $B$ having condition $d_{A, B}(n) \ll f(n)$ we have $s_{B}(x) \ll f(x)$. The next theorem tells us that this is sharp for $f(n)=n^{1 / 3}$.

Theorem 6. There exist a Sidon set (i.e., $\left.s_{A}=1\right) A \subset \mathbb{N}, A=\left\{a_{1}, a_{2}, \ldots\right\}$ and a set $B \subset \mathbb{N}, B=\left\{b_{1}, b_{2}, \ldots\right\}$ such that $d_{A, B}(n) \ll n^{1 / 3}$ and $s_{B}(n) \gg n^{1 / 3}$.

If $s_{A}$ is finite then a routine calculation gives that $A(n)=O(\sqrt{n})$. Hence for $d_{A, B}(n)=$ $O(n)$ we get that $B(n)=O(\sqrt{n})$, which implies that $s_{B}(n)=O(\sqrt{n})$. We pose the following question.

Problem 2. Is it true that for every $1 / 3<\alpha<1 / 2$ there exist sets $A, B \subset \mathbb{N}$ such that $s_{A}<\infty, d_{A, B}(n) \ll n^{\alpha}$ and $s_{B}(n) \gg n^{\alpha}$ ?

The following theorem is about the case when both the representation function $R_{A}(n)$ and the distance $d_{A, B}(n)$ are unbounded.

Theorem 7. Let $A, B \subset \mathbb{N}$ such that $s_{A}=\infty, d_{A, B}=\infty$. Then we have

$$
\max \left\{1, \frac{s_{A}\left(x-2 d_{A, B}(x)\right)}{d_{A, B}(x)}\right\} \ll s_{B}(x) \ll s_{A}\left(x+2 d_{A, B}(x)\right) d_{A, B}(x) .
$$

We will prove Theorem 2. from Theorem 7. Starting out from the set of squares $A=\left\{n^{2}: n \geq 1\right\}$ we will show that for any $\varepsilon>0$ arbitrary small, we have $s_{A}(x)>$ $\exp \left((\log 2-\varepsilon) \frac{\log x}{\log \log x}\right)$ for $x \geq x_{0}(\varepsilon)$. This implies Theorem 2. The following three theorems deals with cubes.

Theorem 8. Let $C$ be the set of positive cubes. Then we have $s_{C}(x) \gg \log \log x$.

The next result is a direct consequence of Theorem 7 and Theorem 8 .

Corollary 1. Let us suppose that for a sequence $a_{n}$ we have $a_{n}=n^{3}+o(\log \log n)$. Then $s_{A}=\infty$

In the other direction we have

Theorem 9. There exists a set of positive integers such that $a_{n}=n^{3}+O\left(n^{2.5} \log n\right)$, and $s_{A}<\infty$. 
Problem 3. What conditions on $\alpha$ are needed to ensure that there exists a sequence $a_{n}$ such that $a_{n}=n^{3}+O\left(n^{\alpha}\right)$, and $s_{A}<\infty$ ?

It follows from Theorem 7 that

Corollary 2. Let $s_{A}(x) \sim x^{\alpha}, d_{A, B}(x) \sim x^{\beta}$. Then we have

$$
x^{\max \{0, \alpha-\beta\}} \ll s_{B}(x) \ll x^{\min \{\alpha+\beta, 1\}} .
$$

Problem 4. Let $\alpha, \beta$ be nonnegative real numbers and $\max \{0, \alpha-\beta\} \leq \gamma \leq \min \{\alpha+$ $\beta, 1\}$. What conditions on $\alpha, \beta$ and $\gamma$ are needed to ensure that there exist $A, B \subset \mathbb{N}$ such that $s_{A}(x) \sim x^{\alpha}, d_{A, B}(x) \sim x^{\beta}$ and $s_{B}(x) \sim x^{\gamma}$ ?

\section{Proofs}

Lemma 1. For any subsets $A$ and $B$ of $\mathbb{N}$ we have

$$
\frac{s_{A}\left(x-2 d_{A, B}(x)\right)}{4 d_{A, B}(x)+1} \leq s_{B}(x) \leq s_{A}\left(x+2 d_{A, B}(x)\right)\left(4 d_{A, B}(x)+1\right) .
$$

Proof. Let $n \leq x$. If $b_{i}+b_{j}=n$, then $b_{i} \leq n \leq x$ and $b_{j} \leq n \leq x$. By the definition of $d_{A, B}(x)$ we have

$$
\left|a_{i}-b_{i}\right| \leq \max _{\mathrm{t}: a_{t} \leq b_{i} \text { or } b_{t} \leq b_{i}}\left|a_{t}-b_{t}\right|=d_{A, B}\left(b_{i}\right)
$$

then we have

$$
-d_{A, B}\left(b_{i}\right) \leq a_{i}-b_{i} \leq d_{A, B}\left(b_{i}\right),
$$

thus

$$
b_{i}-d_{A, B}\left(b_{i}\right) \leq a_{i} \leq d_{A, B}\left(b_{i}\right)+b_{i},
$$

which implies

$$
b_{i}-d_{A, B}(x) \leq a_{i} \leq d_{A, B}(x)+b_{i} .
$$

Similarly for $b_{j}$,

$$
b_{j}-d_{A, B}(x) \leq a_{j} \leq d_{A, B}(x)+b_{j} .
$$

It follows that

$$
b_{i}+b_{j}-2 d_{A, B}(x) \leq a_{i}+a_{j} \leq 2 d_{A, B}(x)+b_{j}+b_{i},
$$

thus

$$
n-2 d_{A, B}(x) \leq a_{i}+a_{j} \leq 2 d_{A, B}(x)+n .
$$

Then we have

$$
\begin{gathered}
R_{B}(n) \leq\left|\left\{(i, j): n-2 d_{A, B}(x) \leq a_{i}+a_{j} \leq n+2 d_{A, B}(x), i \leq j\right\}\right| \\
\leq \sum_{n-2 d_{A, B}(x) \leq m \leq n+2 d_{A, B}(x)} R_{A}(m) \leq\left(4 d_{A, B}(x)+1\right) \max _{n-2 d_{A, B}(x) \leq m \leq n+2 d_{A, B}(x)} R_{A}(m) \\
\leq s_{A}\left(x+2 d_{A, B}(x)\right)\left(4 d_{A, B}(x)+1\right),
\end{gathered}
$$


which proves the second inequality of (1). If we replace $x$ by $x-2 d_{A, B}(x)$, and $A$ by $B$ in the second inequality and by using the fact that $d_{A, B}\left(x-2 d_{A, B}(x)\right) \leq d_{A, B}(x)$, we obtain that

$$
\begin{gathered}
\left.s_{A}\left(x-2 d_{A, B}(x)\right) \leq s_{B}\left(x-2 d_{A, B}(x)+2 d_{A, B}\left(x-2 d_{A, B}(x)\right)\right)\left(4 d_{A, B}\left(x-2 d_{A, B}(x)\right)+1\right)\right) \\
\leq s_{B}(x)\left(4 d_{A, B}(x)+1\right)
\end{gathered}
$$

which proves the first inequality in (1).

Proof of the first part of Theorem 3. It is clear that there exists an $x_{0}$ such that if $x \geq x_{0}$ then $s_{A}(x)=s_{A}, s_{B}(x)=s_{B}, d_{A, B}(x)=d_{A, B}$. If we choose $x=x_{0}+2 d_{A, B}$ it follows from (1) that

$\frac{s_{A}\left(x_{0}+2 d_{A, B}-2 d_{A, B}(x)\right)}{4 d_{A, B}\left(x_{0}+2 d_{A, B}\right)+1} \leq s_{B}\left(x_{0}+2 d_{A, B}\right) \leq s_{A}\left(x_{0}+4 d_{A, B}(x)\right)\left(4 d_{A, B}\left(x_{0}+2 d_{A, B}\right)+1\right)$,

thus

$$
\frac{s_{A}}{4 d_{A, B}+1} \leq s_{B} \leq\left(4 d_{A, B}+1\right) s_{A},
$$

which proves the first part of Theorem 3 .

Proof of the first part of Theorem 4. The first and the third inequality follows from Lemma 1 immediately. We prove the second inequality by contradiction. Assume that

$$
\liminf _{x \rightarrow \infty} \frac{s_{B}(x)}{s_{A}\left(x-2 d_{A, B}\right)}=\alpha, \quad \limsup _{x \rightarrow \infty} \frac{s_{B}(x)}{s_{A}\left(x+2 d_{A, B}\right)}=\beta,
$$

where $\alpha>\beta$. Then for any $\varepsilon>0$ there exist an $x_{0}$ such that for $x \geq x_{0}$ we have $s_{B}(x)>(\alpha-\varepsilon) s_{A}\left(x-2 d_{A, B}\right)$ and $s_{B}(x)<(\beta+\varepsilon) s_{A}\left(x+2 d_{A, B}\right)$. Let $x_{0} \leq N \leq M$. Then we have

$$
\sum_{N \leq x \leq M}(\alpha-\varepsilon) s_{A}\left(x-2 d_{A, B}\right)<\sum_{N \leq x \leq M}(\beta+\varepsilon) s_{A}\left(x+2 d_{A, B}\right),
$$

thus

$$
\begin{gathered}
\sum_{N+2 d_{A, B} \leq x \leq M-2 d_{A, B}}(\alpha-\beta-2 \varepsilon) s_{A}(x) \\
\leq \sum_{M-2 d_{A, B}<x \leq M+2 d_{A, B}}(\beta+\varepsilon) s_{A}(x) \\
\leq 4(\beta+\varepsilon) s_{A}\left(M+2 d_{A, B}\right) d_{A, B} .
\end{gathered}
$$

Let $\varepsilon=\frac{\alpha-\beta}{3}$. We may choose $N$ such that

$$
s_{A}(N) \geq \frac{4(\beta+\varepsilon) 4 d_{A, B}}{\alpha-\beta} .
$$

Then we have

$$
\sum_{N+2 d_{A, B}<x \leq M-2 d_{A, B}}(\alpha-\beta-2 \varepsilon) s_{A}(x) \geq \frac{\alpha-\beta}{3}\left(M-N-4 d_{A, B}\right) s_{A}(N) \geq
$$




$$
\frac{\alpha-\beta}{3} \cdot \frac{4(\beta+\varepsilon) 4 d_{A, B}}{\alpha-\beta}\left(M-N-4 d_{A, B}\right)=\frac{4}{3}(\beta+\varepsilon) 4\left(M-N-4 d_{A, B}\right) d_{A, B} .
$$

It follows from (2) that

$$
\frac{4}{3}(\beta+\varepsilon) 4\left(M-N-4 d_{A, B}\right) d_{A, B} \leq 4(\beta+\varepsilon)\left(M+2 d_{A, B}\right) d_{A, B}
$$

thus we have

$$
\frac{4}{3}\left(M-N-4 d_{A, B}\right) \leq M+2 d_{A, B},
$$

which is a contradiction if $N$ is fixed and $M$ is large enough.

Proof of Theorem 7. Theorem 7. follows from Lemma 1 immediately.

Proof of Theorem 2. Let $A$ be the set of positive squares. Consider the sequence primes $q_{1}<q_{2}<\ldots$ where every $q_{i} \equiv 1 \bmod 4$. Define

$$
Q_{k}=\prod_{i=1}^{k} q_{i}
$$

Let $Q_{K}$ denote the maximal $Q_{k}$ not exceeding $x$. It is easy to see from the well known formula about the number of representations of a positive integer as the sum of two squares [23] that $R_{A}\left(Q_{K}\right)=2^{K-1}$. The well known formula for primorial [27] implies that

$$
Q_{K}=e^{(1+o(1)) K \log K}
$$

It follows that

$$
R_{A}\left(Q_{K}\right)=e^{(\log 2+o(1)) \frac{\log Q_{K}}{\log \log Q_{K}}}
$$

It is clear that

$$
\frac{\log \left(Q_{k+1}\right)}{\log \left(Q_{k}\right)} \rightarrow 1
$$

as $k \rightarrow \infty$. Thus we have

$$
s_{A}(x) \geq e^{(\log 2+o(1)) \frac{\log x}{\log \log x}}
$$

We apply Theorem 7. If $b_{n}=n^{2}+O\left(e^{(\log 2-\varepsilon) \frac{\log x}{\log \log x}}\right)$, then $d_{A, B}(x)=O\left(e^{(\log 2-\varepsilon) \frac{\log x}{\log \log x}}\right)$. Then by Theorem 7 . we have

$$
s_{B}(x) \gg \frac{s_{A}\left(x-2 d_{A, B}(x)\right)}{d_{A, B}(x)} \geq \frac{e^{(\log 2+o(1)) \frac{\log x}{\log \log x}}}{e^{(\log 2-\varepsilon) \frac{\log x}{\log \log x}}}=e^{(\varepsilon+o(1)) \frac{\log x}{\log \log x}},
$$

which implies that $s_{B}=\infty$, thus the function $R_{B}(n)$ is unbounded.

To prove the second part of Theorem 3. and Theorem 4. we need the following Lemma.

Lemma 2. Let $a(n), b(n), d(n)$ be monotone increasing sequences of positive integers satisfying

$$
\frac{a(n)}{4 d(n)+1} \leq b(n) \leq a(n)(4 d(n)+1) .
$$


We put

$$
T(n)=\sum_{k \leq n} \max \{a(k), b(k)\}
$$

Let $c_{n}=d(n)\left(2+10^{n+T(n)}\right)$. Then there exist sets $A, B \subset \mathbb{N}$ such that the following holds.

(i) $s_{A}\left(c_{n}-2 d(n)\right)=s_{A}\left(c_{n}+2 d(n)\right)=a(n)$,

(ii) $s_{B}\left(c_{n}-2 d(n)\right)=s_{B}\left(c_{n}+2 d(n)\right)=b(n)$,

(iii) $d_{A, B}\left(c_{n}\right)=d(n)$,

(iv) $A\left(c_{n}\right)=B\left(c_{n}\right)=2 T(n) \geq 2 n$.

Proof. We give a construction for the sets $A, B$, which built up by blocks. Let $A=$ $\cup_{n=1}^{\infty} A^{(n)}$, and $B=\cup_{n=1}^{\infty} B^{(n)}$, where

$$
\begin{aligned}
& A^{(n)}=\left\{a_{i}^{(n)}: 1 \leq i \leq 2 \max \{a(n), b(n)\}\right\} . \\
& B^{(n)}=\left\{b_{i}^{(n)}: 1 \leq i \leq 2 \max \{a(n), b(n)\}\right\} .
\end{aligned}
$$

Assume that $\max \{a(n), b(n)\}=a(n)$. Then let

$$
a_{i}^{(n)}= \begin{cases}d(n) 10^{n-1+i+T(n-1)}, & \text { if } 1 \leq i \leq a(n) \\ d(n) 10^{n+T(n)}-d(n) 10^{n-1+i-a(n)+T(n-1)}, & \text { if } a(n)+1 \leq i \leq 2 a(n),\end{cases}
$$

therefore $a_{i}^{(n)}=c_{n}-2 d(n)-a_{i-a(n)}^{(n)}$ for $a(n)+1 \leq i \leq 2 a(n)$. It is easy to see that for any $1 \leq i \leq a(m)$ and $a \in A, a<a_{i}^{(m)}$ we have

$$
10 a \leq a_{i}^{(m)}
$$

Let

$$
b_{i}^{(n)}=\left\{\begin{array}{cl}
d(n) 10^{n-1+i+T(n-1)}-d(n)-1+\left\lceil\frac{i}{2 b(n)}\right\rceil, & \text { if } 1 \leq i \leq a(n) \\
d(n) 10^{n+T(n)}-d(n) 10^{n-1+i-a(n)+T(n)} & \\
-d(n)-1+\left\lceil\frac{i-a(n)+b(n)}{2 b(n)}\right\rceil, & \text { if } a(n)+1 \leq i \leq 2 a(n) .
\end{array}\right.
$$

Assume that $\max \{a(n), b(n)\}=b(n)$. Then let

$$
a_{i}^{(n)}= \begin{cases}d(n) 10^{n-1+i+T(n-1)}-d(n)-1+\left\lceil\frac{i}{2 a(n)}\right\rceil, & \text { if } 1 \leq i \leq b(n) \\ d(n) 10^{n+T(n)}-d(n) 10^{n-1+i-b(n)+T(n-1)}-d(n)-1+\left\lceil\frac{i-b(n)+a(n)}{2 a(n)}\right\rceil, & \text { if } b(n)+1 \leq i \leq 2 b(n),\end{cases}
$$

and

$$
b_{i}^{(n)}= \begin{cases}d(n) 10^{n-1+i+T(n-1)}, & \text { if } 1 \leq i \leq b(n) \\ d(n) 10^{n+T(n)}-d(n) 10^{n-1+i-b(n)+T(n-1)}, & \text { if } b(n)+1 \leq i \leq 2 b(n) .\end{cases}
$$

It is easy to see that for any $1 \leq i \leq b(m)$ and $a \in A, a<a_{i}^{(m)}$ we have

$$
10 a \leq a_{i}^{(m)} .
$$


In the next step we prove that the above sets $A$ and $B$ satisfy the Lemma.

First we prove $(i)$. To do this we show that both $s_{A}\left(c_{n}+2 d(n)\right) \geq a(n)$ and $s_{A}\left(c_{n}+\right.$ $2 d(n)) \leq a(n)$ hold. In the first case assume that $\max \{a(n), b(n)\}=a(n)$. It is clear from the definition of $a_{i}^{(n)}$ that $a_{i}^{(n)}+a_{i+a(n)}^{(n)}=d(n) 10^{n+T(n)}=c_{n}-2 d(n)$ for $1 \leq$ $i \leq a(n)$. Therefore, $R_{A}\left(d(n) 10^{n+T(n)}\right)=R_{A}\left(c_{n}-2 d(n)\right) \geq a(n)$. Thus we have $s_{A}\left(c_{n}+2 d(n)\right) \geq a(n)$. In the second case we assume that $\max \{a(n), b(n)\}=b(n)$. Now we have $a_{i}^{(n)}+a_{i+b(n)}^{(n)}=d(n) 10^{n+T(n)}-2 d(n)=c_{n}-4 d(n)$ for $1 \leq i \leq a(n)$, which implies that $R_{A}\left(c_{n}-4 d(n)\right) \geq a(n)$ and so $s_{A}\left(c_{n}+2 d(n)\right) \geq a(n)$. It remains to prove that $s_{A}\left(c_{n}+2 d(n)\right) \leq a(n)$. Since $d(n)$ is monotone increasing, if $\max \{a(n), b(n)\}=a(n)$ then $a_{1}^{(n+1)}=d(n+1) 10^{n+1+T(n)}>c_{n}+2 d(n)=d(n) 10^{n+T(n)}+4 d(n)$. On the other hand, if $\max \{a(n), b(n)\}=b(n)$ then $a_{1}^{(n+1)}=d(n+1) 10^{n+1+T(n+1)}-d(n+1)>c_{n}+2 d(n)=$ $d(n) 10^{n+T(n)}+4 d(n)$. It is enough to show that $R_{A}\left(a_{s}+a_{t}\right) \leq a(n)$ for any fixed

$$
a_{s}, a_{t} \in \cup_{i=1}^{n} A^{(i)} \text {. }
$$

In the next we give an upper estimation to the number of pairs $(u, v)$ such that $a_{s}+a_{t}=$ $a_{u}+a_{v}$, where $a_{u}, a_{v} \in A$, and we may suppose that $a_{s} \geq a_{u} \geq a_{v} \geq a_{t}$, and $a_{s} \in A^{(m)}$ for some $m \leq n$. We distinguish two cases.

In the first case assume that $\max \{a(m), b(m)\}=a(m)$. We will prove that if $a_{s}+a_{t}=$ $a_{u}+a_{v}$ is a nontrivial solution, i.e., $R_{A}\left(a_{s}+a_{t}\right) \geq 2$, then

$$
a_{s}+a_{t}=d(m) 10^{m+T(m)} .
$$

We have five subcases depending on how many of $a_{s}, a_{t}, a_{u}, a_{v}$ are selected from the set $\left\{a_{1+10^{m}}^{(m)}, a_{2+10^{m}}^{(m)}, \ldots, a_{10^{m}}^{(m)}+10^{m}\right\}$.

If none of them are selected from the set $\left\{a_{1+10^{m}}^{(m)}, a_{2+10^{m}}^{(m)}, \ldots, a_{10^{m}+10^{m}}^{(m)}\right\}$, then $a_{j}^{(m)}+$ $a_{t}=a_{u}+a_{v}$. It follows from (3) that $a_{u}=a_{j}^{(m)}$, thus $a_{s}+a_{t}=a_{u}+a_{v}$ must be a trivial solution.

If one of the terms $a_{s}, a_{t}, a_{u}, a_{v}$ are selected from the set $\left\{a_{1+10^{m}}^{(m)}, a_{2+10^{m}}^{(m)}, \ldots, a_{10^{m}+10^{m}}^{(m)}\right\}$, that is, $a_{s}=a_{j}^{(m)}$, for some $a(m)<j \leq 2 a(m)$ then it is clear that $a_{j}^{(m)}+a_{t}>a_{u}+a_{v}$, a contradiction.

If two of the terms $a_{s}, a_{t}, a_{u}, a_{v}$ are selected from the set $\left\{a_{1+10^{m}}^{(m)}, a_{2+10^{m}}^{(m)}, \ldots, a_{10^{m}+10^{m}}^{(m)}\right\}$, then let $a_{s}=a_{j}^{(m)}$ and $a_{u}=a_{k}^{(m)}$, where $a(m)<j \leq k \leq 2 a(m)$. Then we have

$$
c_{m}-2 d(m)-a_{j-a(m)}^{(m)}+a_{t}=c_{m}-2 d(m)-a_{k-a(m)}^{(m)}+a_{v} .
$$

Thus we have

$$
a_{k-a(m)}^{(m)}+a_{t}=a_{j-a(m)}^{(m)}+a_{v} .
$$

If $k=j$ then $a_{s}+a_{t}=a_{u}+a_{v}$ is a trivial solution. If $j<k$ then it follows from (3) that $a_{v}=a_{k-a(m)}^{(m)}$ and $a_{t}=a_{j-a(m)}^{(m)}$, therefore $a_{s}+a_{t}=a_{j}^{(m)}+a_{j-a(m)}^{(m)}=d(m) 10^{m+T(m)}$.

If three of the terms $a_{s}, a_{t}, a_{u}, a_{v}$ are selected from the set $\left\{a_{1+10^{m}}^{(m)}, a_{2+10^{m}}^{(m)}, \ldots, a_{10^{m}+10^{m}}^{(m)}\right\}$, then let $a_{s}=a_{j}^{(m)}$ and $a_{u}=a_{k}^{(m)}$, where $a(m)<j \leq k \leq 2 a(m)$. Then we have

$$
a_{k-a(m)}^{(m)}+a_{t}=a_{j-a(m)}^{(m)}+a_{v},
$$


where only one term is selected from the set $\left\{a_{1+10^{m}}^{(m)}, a_{2+10^{m}}^{(m)}, \ldots, a_{10^{m}+10^{m}}^{(m)}\right\}$, which is absurd.

If four of the terms $a_{s}, a_{t}, a_{u}, a_{v}$ are selected from the set $\left\{a_{1+10^{m}}^{(m)}, a_{2+10^{m}}^{(m)}, \ldots, a_{10^{m}+10^{m}}^{(m)}\right\}$, then let $a_{s}=a_{j}^{(m)}, a_{u}=a_{k}^{(m)}, a_{v}=a_{l}^{(m)}$ and $a_{t}=a_{q}^{(m)}$ where $a(m)<j \leq k \leq l \leq q \leq$ $2 a(m)$. Then

$$
a_{k-a(m)}^{(m)}+a_{l-a(m)}^{(m)}=a_{j-a(m)}^{(m)}+a_{q-a(m)}^{(m)},
$$

which must be a trivial solution.

We have $a(m)$ elements of the set $A$ in the interval

$$
\left[\frac{d(m)}{2} 10^{m+T(m)}, d(m) 10^{m+T(m)}\right]
$$

therefore $R_{A}\left(a_{s}+a_{t}\right) \leq a(m) \leq a(n)$.

In the second case assume that $\max \{a(m), b(m)\}=b(m)$. In this case we prove that the nontrivial equation $a_{s}+a_{t}=a_{u}+a_{v}$ implies the existence of the integers $1 \leq j \leq k \leq b(m)$ such that $a_{s}=a_{j}^{(m)}, a_{t}=a_{j-b(m)}^{(m)}, a_{u}=a_{k}^{(m)}$ and $a_{v}=a_{k-b(m)}^{(m)}$.

Then we have five subcases as above.

If none of them are selected from the set $\left\{a_{1+10^{m}}^{(m)}, a_{2+10^{m}}^{(m)}, \ldots, a_{10^{m}+10^{m}}^{(m)}\right\}$, then $a_{s}+a_{t}=$ $a_{u}+a_{v}$, where $a_{s}=a_{j}^{(m)}$ and $1 \leq j \leq b(m)$. It follows from (4) that $a_{u}=a_{j}^{(m)}$, thus $a_{s}+a_{t}=a_{u}+a_{v}$ must be a trivial solution.

If one of the terms $a_{s}, a_{t}, a_{u}, a_{v}$ are selected from the set $\left\{a_{1+10^{m}}^{(m)}, a_{2+10^{m}}^{(m)}, \ldots, a_{10^{m}+10^{m}}^{(m)}\right\}$, that is, $a_{s}=a_{j}^{(m)}$, for some $b(m)<j \leq 2 b(m)$ then it is clear that $a_{j}^{(m)}+a_{t}>a_{u}+a_{v}$, a contradiction.

If two of the terms $a_{s}, a_{t}, a_{u}, a_{v}$ are selected from the set $\left\{a_{1+10^{m}}^{(m)}, a_{2+10^{m}}^{(m)}, \ldots, a_{10^{m}+10^{m}}^{(m)}\right\}$, then let $a_{s}=a_{j}^{(m)}$ and $a_{u}=a_{k}^{(m)}$, where $b(m)<j \leq k \leq 2 b(m)$. Then the equation $a_{s}+a_{t}=a_{u}+a_{v}$ means that

$$
\begin{aligned}
& d(m) 10^{m+T(m)}-d(m) 10^{m-1+j-b(m)+T(m-1)}-d(m)-1+\left\lceil\frac{j-b(m)+a(m)}{2 a(m)}\right\rceil+a_{t} \\
= & d(m) 10^{m+T(m)}-d(m) 10^{m-1+k-b(m)+T(m-1)}-d(m)-1+\left\lceil\frac{k-b(m)+a(m)}{2 a(m)}\right\rceil+a_{v},
\end{aligned}
$$

that is

$$
\begin{gathered}
d(m) 10^{m-1+k-b(m)+T(m-1)}+a_{t} \\
=d(m) 10^{m-1+j-b(m)+T(m-1)}+a_{v}+\left\lceil\frac{k-b(m)+a(m)}{2 a(m)}\right\rceil-\left\lceil\frac{j-b(m)+a(m)}{2 a(m)}\right\rceil,
\end{gathered}
$$

therefore,

$$
\begin{gathered}
d(m) 10^{m-1+k-b(m)+T(m-1)}-d(m)-1+\left\lceil\frac{k-b(m)}{2 a(m)}\right\rceil+a_{t} \\
=d(m) 10^{m-1+j-b(m)+T(m-1)}-d(m)-1+\left\lceil\frac{j-b(m)}{2 a(m)}\right\rceil+a_{v} \\
+\left\lceil\frac{k-b(m)}{2 a(m)}\right\rceil-\left\lceil\frac{j-b(m)}{2 a(m)}\right\rceil+\left\lceil\frac{k-b(m)+a(m)}{2 a(m)}\right\rceil-\left\lceil\frac{j-b(m)+a(m)}{2 a(m)}\right\rceil,
\end{gathered}
$$


i.e.,

$$
\begin{aligned}
a_{k-b(m)}^{(m)}+a_{t} & =a_{j-b(m)}^{(m)}+a_{v}+\left\lceil\frac{k-b(m)}{2 a(m)}\right\rceil+\left\lceil\frac{k-b(m)+a(m)}{2 a(m)}\right\rceil \\
& -\left(\left\lceil\frac{j-b(m)}{2 a(m)}\right\rceil+\left\lceil\frac{j-b(m)+a(m)}{2 a(m)}\right\rceil\right) .
\end{aligned}
$$

If $k=j$ then $a_{s}+a_{t}=a_{u}+a_{v}$ is a trivial solution. Since $b(m) \leq a(m)(4 d(m)+1)$ and $b(m)<j \leq k \leq 2 b(m)$, if $j<k$ then we have

$$
1 \leq\left\lceil\frac{k-b(m)}{2 a(m)}\right\rceil \leq\left\lceil\frac{b(m)}{2 a(m)}\right\rceil \leq\left\lceil\frac{(4 d(m)+1) a(m)}{2 a(m)}\right\rceil=2 d(m)+1
$$

and

$$
1 \leq\left\lceil\frac{k-b(m)+a(m)}{2 a(m)}\right\rceil \leq\left\lceil\frac{(4 d(m)+1) a(m)+a(m)}{2 a(m)}\right\rceil=2 d(m)+1 .
$$

It follows from (4) that $a_{v}=a_{k-b(m)}^{(m)}$ and by the definition of $a_{j-b(m)}^{(m)}=d(m) 10^{m-1+j-b(m)+T(m-1)}$ it follows that $a_{t}=a_{j-b(m)}^{(m)}$. Fix the elements $a_{s}$ and $a_{t}$. The equation $a_{s}+a_{t}=a_{u}+a_{v}$ can be written in the form $a_{j}^{(m)}+a_{j-b(m)}^{(m)}=a_{k}^{(m)}+a_{k-b(m)}^{(m)}$.

If three of the terms $a_{s}, a_{t}, a_{u}, a_{v}$ are selected from the set $\left\{a_{1+10^{m}}^{(m)}, a_{2+10^{m}}^{(m)}, \ldots, a_{10^{m}+10^{m}}^{(m)}\right\}$, then let $a_{s}=a_{j}^{(m)}, a_{u}=a_{k}^{(m)}$ and $a_{v}=a_{l}^{(m)}$, where $b(m)<j \leq k \leq l \leq 2 b(m)$. Then we have

$$
a_{j}^{(m)}+a_{t}<a_{k}^{(m)}+a_{l}^{(m)},
$$

where only one term is selected from the set $\left\{a_{1+10^{m}}^{(m)}, a_{2+10^{m}}^{(m)}, \ldots, a_{10^{m}+10^{m}}^{(m)}\right\}$, which is absurd as we have seen earlier.

If four of the terms $a_{s}, a_{t}, a_{u}, a_{v}$ are selected from the set $\left\{a_{1+10^{m}}^{(m)}, a_{2+10^{m}}^{(m)}, \ldots, a_{10^{m}+10^{m}}^{(m)}\right\}$, then let $a_{s}=a_{j}^{(m)}, a_{u}=a_{k}^{(m)}, a_{v}=a_{l}^{(m)}$ and $a_{t}=a_{m}^{(m)}$ where $b(m)<j \leq k \leq l \leq q \leq$ $2 b(m)$. Then by definition the equation $a_{j}^{(m)}+a_{q}^{(m)}=a_{k}^{(m)}+a_{l}^{(m)}$ means that

$$
\begin{aligned}
& d(m) 10^{m+T(m)}-d(m) 10^{m-1+j-b(m)+T(m-1)}-d(m)-1+\left\lceil\frac{j-b(m)+a(m)}{2 a(m)}\right\rceil \\
+ & d(m) 10^{m+T(m)}-d(m) 10^{m-1+q-b(m)+T(m-1)}-d(m)-1+\left\lceil\frac{q-b(m)+a(m)}{2 a(m)}\right\rceil \\
= & d(m) 10^{m+T(m)}-d(m) 10^{m-1+k-b(m)+T(m-1)}-d(m)-1+\left\lceil\frac{k-b(m)+a(m)}{2 a(m)}\right\rceil \\
+ & d(m) 10^{m+T(m)}-d(m) 10^{m-1+l-b(m)+T(m-1)}-d(m)-1+\left\lceil\frac{l-b(m)+a(m)}{2 a(m)}\right\rceil .
\end{aligned}
$$

Therefore,

$$
\begin{aligned}
& d(m) 10^{m-1+j-b(m)+T(m-1)}-\left\lceil\frac{j-b(m)+a(m)}{2 a(m)}\right\rceil+d(m) 10^{m-1+q-b(m)+T(m-1)}-\left\lceil\frac{q-b(m)+a(m)}{2 a(m)}\right\rceil \\
& =d(m) 10^{m-1+k-b(m)+T(m-1)}-\left\lceil\frac{k-b(m)+a(m)}{2 a(m)}\right\rceil+d(m) 10^{m-1+l-b(m)+T(m-1)}-\left\lceil\frac{l-b(m)+a(m)}{2 a(m)}\right\rceil,
\end{aligned}
$$


which implies that $q=l$ and $j=k$, a trivial solution.

If $R_{A}\left(a_{s}+a_{t}\right)>1$ then for the nontrivial solution $a_{s}+a_{t}=a_{u}+a_{v}$ we have integers $1 \leq j \leq k \leq b(m)$ such that $a_{s}=a_{j}^{(m)} a_{t}=a_{j-b(m)}^{(m)}, a_{u}=a_{k}^{(m)}, a_{v}=a_{k-b(m)}^{(m)}$.

By the definition we have

$$
\begin{gathered}
d(m) 10^{m+T(m)}-d(m) 10^{m-1+j-b(m)+T(m-1)}-d(m)-1+\left\lceil\frac{j-b(m)+a(m)}{2 a(m)}\right\rceil \\
+d(m) 10^{m-1+j-b(m)+T(m-1)}-d(m)-1+\left\lceil\frac{j-b(m)}{2 a(m)}\right\rceil \\
=d(m) 10^{m+T(m)}-d(m) 10^{m-1+k-b(m)+T(m-1)}-d(m)-1+\left\lceil\frac{k-b(m)+a(m)}{2 a(m)}\right\rceil \\
+d(m) 10^{m-1+k-b(m)+T(m-1)}-d(m)-1+\left\lceil\frac{k-b(m)}{2 a(m)}\right\rceil .
\end{gathered}
$$

Thus we have

$$
\left\lceil\frac{k-b(m)+a(m)}{2 a(m)}\right\rceil+\left\lceil\frac{k-b(m)}{2 a(m)}\right\rceil=\left\lceil\frac{j-b(m)+a(m)}{2 a(m)}\right\rceil+\left\lceil\frac{j-b(m)}{2 a(m)}\right\rceil .
$$

Let $j=\left(l_{1}-2\right) a(m)+h_{1}$ and $k=\left(l_{2}-2\right) a(m)+h_{2}$, where $1 \leq h_{1}, h_{2} \leq a(m)$. Then we have

$$
\left\lceil\frac{\left(l_{1}-2\right) a(m)+h_{1}}{2 a(m)}\right\rceil+\left\lceil\frac{\left(l_{1}-1\right) a(m)+h_{1}}{2 a(m)}\right\rceil=\left\lceil\frac{\left(l_{2}-2\right) a(m)+h_{2}}{2 a(m)}\right\rceil+\left\lceil\frac{\left(l_{2}-1\right) a(m)+h_{2}}{2 a(m)}\right\rceil,
$$

hence

$$
\left\lceil\frac{l_{1}-1}{2}\right\rceil+\left\lceil\frac{l_{1}}{2}\right\rceil=\left\lceil\frac{l_{2}-1}{2}\right\rceil+\left\lceil\frac{l_{2}}{2}\right\rceil,
$$

that is $l_{1}=l_{2}$, which implies that the number of suitable pairs $(u, v)$ is at most $a(m) \leq$ $a(n)$.

Changing the role of $a(n)$ and $b(n)$, the proof of (ii) is the same as the proof (i) thus we omit it and leave the details to the reader.

Now we prove (iii). Assume that $\max \{a(m), b(m)\}=a(m)$, where $m \leq n$. Then if $1 \leq i \leq a(m)$, then

$$
a_{i}^{(m)}-b_{i}^{(m)}=d(m)+1-\left\lceil\frac{i}{2 b(m)}\right\rceil .
$$

As $1 \leq i \leq a(m) \leq(4 d(m)+1) b(m)$, then we have

$$
1 \leq\left\lceil\frac{i}{2 b(m)}\right\rceil \leq\left\lceil\frac{(4 d(m)+1) b(m)}{2 b(m)}\right\rceil=2 d(m)+1
$$

then $-d(m) \leq a_{i}^{(m)}-b_{i}^{(m)} \leq d(m)$. If $a(m)<i \leq 2 a(m)$, then

$$
a_{i}^{(m)}-b_{i}^{(m)}=d(m)+1-\left\lceil\frac{i-a(m)+b(m)}{2 b(m)}\right\rceil .
$$

Since $a(m) \leq(4 d(m)+1) b(m)$, then

$$
1 \leq\left\lceil\frac{i-a(m)+b(m)}{2 b(m)}\right\rceil \leq\left\lceil\frac{a(m)+b(m)}{2 b(m)}\right\rceil \leq\left\lceil\frac{(4 d(m)+1) b(m)+b(m)}{2 b(m)}\right\rceil=2 d(m)+1,
$$


thus $-d(m) \leq a_{i}^{(m)}-b_{i}^{(m)} \leq d(m)$. As $d(m)$ is monotonous increasing and $\left|a_{1}^{(m)}-b_{1}^{(m)}\right|=$ $d(m)$, it follows (iii). If $\max \{a(m), b(m)\}=b(m)$, changing the role of $a(m)$ and $b(m)$, the argument is the same.

The statement (iv) follows easily from the construction. By the definition of $A^{(n)}$ it is clear that it has at least two elements. Therefore, up to $c_{n}$ the set $A$ has at least $2 n$ elements. The same argument works for the set $B$ as well.

Proof of the second part of Theorem 3. If we put $a(n)=a, b(n)=b, d(n)=d$ in Lemma 2, the Theorem follows immediately.

Proof of the second part of Theorem 4. Let us suppose that the monotone increasing sequences $\left\{u_{n}\right\}$ and $\left\{v_{n}\right\}$ satisfy

$$
\lim _{n \rightarrow \infty} \frac{u_{n}}{v_{n}}=\alpha
$$

and

$$
\lim _{n \rightarrow \infty} \frac{u_{n+1}}{v_{n}}=\beta .
$$

Let $b(2 n-1)=u_{n}, b(2 n)=u_{n+1}, a(2 n-1)=a(2 n)=v_{n}$, and $d(n)=d_{A, B}$ in Lemma 2 . then by the construction we have

$$
\frac{s_{B}\left(c_{2 n-1}\right)}{s_{A}\left(c_{2 n-1}-2 d_{A, B}\right)}=\frac{u_{n}}{v_{n}} \rightarrow \alpha
$$

and

$$
\frac{s_{B}\left(c_{2 n}\right)}{s_{A}\left(c_{2 n}+2 d_{A, B}\right)}=\frac{u_{n+1}}{v_{n}} \rightarrow \beta
$$

If $c_{2 n-1} \leq k \leq c_{2 n}$, then $s_{A}\left(c_{2 n-1}-2 d_{A, B}\right)=s_{A}\left(k-2 d_{A, B}\right)=s_{A}\left(k+2 d_{A, B}\right)=s_{A}\left(c_{2 n}+\right.$ $\left.2 d_{A, B}\right)=v_{n}$. It follows that

$$
\alpha \leftarrow \frac{s_{B}\left(c_{2 n-1}\right)}{s_{A}\left(c_{2 n-1}-2 d_{A, B}\right)} \leq \frac{s_{B}(k)}{s_{A}\left(k-2 d_{A, B}\right)}=\frac{s_{B}(k)}{s_{A}\left(k+2 d_{A, B}\right)} \leq \frac{s_{B}\left(c_{2 n}\right)}{s_{A}\left(c_{2 n}+2 d_{A, B}\right)} \rightarrow \beta .
$$

If $c_{2 n} \leq k \leq c_{2 n+1}$, then $s_{B}\left(c_{2 n}-2 d_{A, B}\right)=s_{B}(k)=s_{B}\left(c_{2 n+1}+2 d_{A, B}\right)=u_{n+1}$. It follows that

$$
\alpha \leftarrow \frac{s_{B}\left(c_{2 n+1}\right)}{s_{A}\left(c_{2 n+1}-2 d_{A, B}\right)} \leq \frac{s_{B}(k)}{s_{A}\left(k+2 d_{A, B}\right)} \leq \frac{s_{B}(k)}{s_{A}\left(k-2 d_{A, B}\right)} \leq \frac{s_{B}\left(c_{2 n}\right)}{s_{A}\left(c_{2 n}+2 d_{A, B}\right)} \rightarrow \beta,
$$

which completes the proof.

Proof of Theorem 5. First assume that $b$ is a positive integer. Let $a(n)=a$. Without loss of generality we may assume that $a \leq b$. Let

$$
b(n)= \begin{cases}a, & \text { if } f(n)<\left\lceil\frac{b}{a}\right\rceil \\ b, & \text { if } f(n) \geq\left\lceil\frac{b}{a}\right\rceil,\end{cases}
$$

and let $d(n)=f(n)$ in Lemma 2. Since $A\left(c_{n}\right) \geq 2 n, d_{A, B}\left(c_{n}\right)=d(n)=f(n)$, then if $k \leq 2 n$, this implies that $\left|a_{k}-b_{k}\right| \leq f(n)$, therefore $\left|a_{n}-b_{n}\right| \leq f(n)$. 
On the other hand, if $b=+\infty$, then let $a(n)=a, b(n)=a f(n), d(n)=f(n)$ in Lemma 2. It follows that $s_{B}=+\infty, s_{A}=a, A\left(c_{n}\right) \geq 2 n, d_{A, B}\left(c_{n}\right)=d(n)=f(n)$. This implies that $\left|a_{n}-b_{n}\right| \leq f(n)$.

Proof of Theorem 6. We give a construction for the sets $A$ and $B$ recursively. Define the sets $A^{(m)}$ and $B^{(m)}$ in the following way. Fix a nonnegative integer $m$ and we will choose the distinct positive integers $200 \cdot 1000^{m} \leq b_{1}^{(m)}, \ldots, b_{10^{m}}^{(m)} \leq 300$. $1000^{m}$. Define $b_{i+10^{m}}^{(m)}$ by $b_{i+10^{m}}^{(m)}=1000^{m+1}-b_{i}^{(m)}$, where $1 \leq i \leq 10^{m}$. Let $B^{(m)}=$ $\left\{b_{1}^{(m)}, \ldots, b_{10^{m}}^{(m)}, b_{10^{m}+1}^{(m)}, \ldots, b_{10^{m}+10^{m}}^{(m)}\right\}$. It is easy to see that $R_{B}\left(1000^{m+1}\right) \geq 10^{m} \gg$ $\left(1000^{m+1}\right)^{1 / 3}$. This implies that $s_{B}(n) \gg n^{1 / 3}$. Now we define the sets $A^{(m)}$ in the following way. Let $a_{i}^{(m)}=b_{i}^{(m)}+i$, and $a_{i+10^{m}}^{(m)}=b_{i+10^{m}}^{(m)}$, where $1 \leq i \leq 10^{m}$. Let $A^{(m)}=\left\{a_{1}^{(m)}, \ldots, a_{10^{m}}^{(m)}, a_{10^{m}+1}^{(m)}, \ldots, a_{10^{m}+10^{m}}^{(m)}\right\}$. Define the sets $A=\cup_{m=1}^{\infty} A^{(m)}$ and $B=$ $\cup_{m=1}^{\infty} B^{(m)}$ and let $A=\left\{a_{1}, a_{2}, \ldots\right\}, a_{1}<a_{2}<\ldots$ and $B=\left\{b_{1}, b_{2}, \ldots\right\}, b_{1}<b_{2}<\ldots$ It is clear that $\left|a_{N}-b_{N}\right| \ll N$ and $a_{N}=\Theta\left(N^{3}\right)$ for every $N$, therefore $d_{A, B}(n) \ll n^{1 / 3}$. In the next step we prove that $A$ may be chosen for a Sidon set. Our strategy is the following. It is clear from the definition that $A$ is built up from blocks. We use the greedy algorithm to construct the set $A$. Assume that we have already constructed the first few blocks, and we have already chosen some elements to obtain the next block. Suppose that this set satisfies the Sidon property. By the definition of a block, $A^{(m)}$ contains two different type of elements, therefore in each step we have to add two new elements to the set had already been constructed. We have to guarantee the Sidon property. Since we have two new elements, we will need an extra condition to ensure that the sum of the two new elements does not destroy the Sidon property either. More formally, let $B_{0}=\{200,800\}$ and $A_{0}=\{201,800\}$ and assume that we have already chosen blocks $A^{(0)}, A^{(1)}, A^{(2)}, \ldots, A^{(m-1)}$ and integers $a_{1}^{(m)}, \ldots, a_{i-1}^{(m)}, a_{10^{m}+1}^{(m)}, \ldots, a_{10^{m}+i-1}^{(m)}$, where $1 \leq i \leq 10^{m}$. For $0 \leq l \leq i-1$, define

$$
\mathcal{A}_{l}^{(m)}=\cup_{t=0}^{m-1} A^{(t)} \cup\left\{a_{1}^{(m)}, \ldots, a_{l}^{(m)}, a_{10^{m}+1}^{(m)}, \ldots, a_{10^{m}+l}^{(m)}\right\} .
$$

We prove by induction on $l$ that the integers $a_{j}^{(m)}, a_{j+10^{m}}^{(m)}$ may be selected such that $\mathcal{A}_{l}^{(m)}$ satisfies the Sidon property, that is $a+a^{\prime} \neq a^{\prime \prime}+a^{\prime \prime \prime}$, where $a, a^{\prime}, a^{\prime \prime}, a^{\prime \prime \prime} \in \mathcal{A}_{l}^{(m)}$ except for the trivial solutions and the extra condition

$$
a+a^{\prime} \neq 1000^{m+1}+j
$$

where $a, a^{\prime} \in \mathcal{A}_{l}^{(m)}$, and for every $l \leq j \leq m$. It is enough to prove that we may add integers $a_{i}^{(m)}$ and $a_{i+10^{m}}^{(m)}$ to the set $\mathcal{A}_{i-1}^{(m)}$ retaining the Sidon property and the extra condition.

$$
a+a^{\prime} \neq 1000^{m+1}+j,
$$

where $a, a^{\prime} \in \mathcal{A}_{i}^{(m)}$, for any $i<j \leq 10^{m}$. In order to guarantee it we distinguish six cases. Let $a, a^{\prime}, a^{\prime \prime} \in \mathcal{A}_{i-1}^{(m)}$, and we put $a_{i}^{(m)}$ and $a_{i+10^{m}}^{(m)}$ to the set $\mathcal{A}_{i-1}^{(m)}$. We have to guarantee that adding new elements $a_{i}^{(m)}$ and $a_{i+10^{m}}^{(m)}$ to the set $\mathcal{A}_{i-1}^{(m)}$ does not destroy its Sidon property i.e.,

$$
a_{i}^{(m)}+a \neq a^{\prime}+a^{\prime \prime}
$$

and

$$
a_{i+10^{m}}^{(m)}+a \neq a^{\prime}+a^{\prime \prime}
$$


and

$$
a_{i}^{(m)}+a_{i+10^{m}}^{(m)} \neq a+a^{\prime},
$$

and

$$
a_{i}^{(m)}+a \neq a_{i+10^{m}}^{(m)}+a^{\prime} .
$$

Moreover, by the extra condition we have

$$
a_{i}^{(m)}+a \neq 1000^{m+1}+j
$$

and

$$
a_{10^{m}+i}^{(m)}+a \neq 1000^{m+1}+j,
$$

where $i<j \leq 10^{m}$.

It is easy to see that the number of elemets of $\mathcal{A}_{i-1}^{(m)}$ is less than $10^{m}+\ldots+1$, thus the number of possibilities to choose triplets $\left(a, a^{\prime}, a^{\prime \prime}\right)$ in (6) and (7) is at most $\left(10^{m}+\ldots+1\right)^{3} \cdot 2<\left(\frac{10}{9} \cdot 10^{m}\right)^{3} \cdot 2$.

In the next step we show that inequality (8) holds. It is clear that $a_{i}^{(m)}+a_{i+10^{m}}^{(m)}=$ $1000^{m+1}+i$ and the extra condition $a+a^{\prime} \neq 1000^{m+1}+j$ for any $a, a^{\prime} \in \mathcal{A}_{i-1}^{(m)}$ and $i-1<j \leq 10^{m}$. In the special case $j=i$ we have $a+a^{\prime} \neq 1000^{m+1}+i$, which proves (8). In equation (9)

$$
a_{i}^{(m)}+a \neq a_{i+10^{m}}^{(m)}+a^{\prime}=1000^{m+1}+i-a_{i}^{(m)}+a^{\prime},
$$

implies that

$$
2 a_{i}^{(m)} \neq 1000^{m+1}+i+a^{\prime}-a .
$$

It is clear that the number of possibilities to choose pairs $\left(a, a^{\prime}\right)$ is at most $(1+\ldots+$ $\left.10^{m}\right)^{2}<\left(\frac{10}{9} 10^{m}\right)^{2}$.

It is easy to see that in inequalities (10) and (11) the number of possibilities to choose pairs $(a, j)$ and is at most $2 \cdot\left(1+\ldots+10^{m}\right) \cdot 10^{m}<\frac{20}{9} \cdot\left(10^{m}\right)^{2}$.

It follows that the number of wrong $a_{i}^{(m)}$ is at most

$$
2\left(\frac{10}{9} 10^{m}\right)^{3}+\left(\frac{10}{9} 10^{m}\right)^{2}+\frac{20}{9} \cdot\left(10^{m}\right)^{2}<100 \cdot 1000^{m} .
$$

This shows that we may choose elements which does not destroy the Sidon property.

Proof of Theorem 8. It is enough to prove that for every $k \geq 1$, there exists an integer $n \leq 100^{100^{k}}$ such that $R_{C}(n) \geq k$. To prove this we use the well-known formulas of Vieta: Let us suppose that

$$
x^{3}+y^{3}=a z^{3},
$$

then we have

$$
\left(x\left(x^{3}+2 y^{3}\right)\right)^{3}+\left(-y\left(2 x^{3}+y^{3}\right)\right)^{3}=a\left(x^{3}-y^{3}\right)^{3} z^{3} .
$$

After repeating we get

$$
\begin{aligned}
& \left(x\left(x^{3}+2 y^{3}\right)\left(x^{3}\left(x^{3}+2 y^{3}\right)^{3}-2 y^{3}\left(2 x^{3}+y^{3}\right)^{3}\right)\right)^{3} \\
+ & \left(y\left(2 x^{3}+y^{3}\right)\left(2 x^{3}\left(x^{3}+2 y^{3}\right)^{3}-y^{3}\left(2 x^{3}+y^{3}\right)^{3}\right)\right)^{3}
\end{aligned}
$$




$$
=a\left(x^{3}-y^{3}\right)^{3}\left(x^{3}\left(x^{3}+2 y^{3}\right)^{3}+y^{3}\left(2 x^{3}+y^{3}\right)^{3}\right)^{3} z^{3} .
$$

We define the sequences $u_{i}, v_{i}$ and $w_{i}$ recursively as follows. Let $u_{1}=4^{k-1}, v_{1}=1$ and $w_{1}=1$ and let

$$
\begin{aligned}
& u_{i+1}=u_{i}\left(u_{i}^{3}+2 v_{i}^{3}\right)\left(u_{i}^{3}\left(u_{i}^{3}+2 v_{i}^{3}\right)^{3}-2 v_{i}^{3}\left(2 u_{i}^{3}+v_{i}^{3}\right)^{3}\right) \\
& v_{i+1}=v_{i}\left(2 u_{i}^{3}+v_{i}^{3}\right)\left(2 u_{i}^{3}\left(u_{i}^{3}+2 v_{i}^{3}\right)^{3}-v_{i}^{3}\left(2 u_{i}^{3}+v_{i}^{3}\right)^{3}\right) \\
& w_{i+1}=\left(u_{i}^{3}-v_{i}^{3}\right)\left(u_{i}^{3}\left(u_{i}^{3}+2 v_{i}^{3}\right)^{3}+v_{i}^{3}\left(2 u_{i}^{3}+v_{i}^{3}\right)^{3}\right) w_{i},
\end{aligned}
$$

for $i=1,2, \ldots, k-1$. Then we have

$$
u_{i}^{3}+v_{i}^{3}=\left(64^{k-1}+1\right) w_{i}^{3}
$$

and $w_{i} \mid w_{k}$. Let $x_{i}=\frac{u_{i}}{w_{i}} w_{k}, y_{i}=\frac{v_{i}}{w_{i}} w_{k}$ and $z_{i}=w_{i}$, for $1 \leq i \leq k$. Hence

$$
x_{i}^{3}+y_{i}^{3}=\left(64^{k-1}+1\right) w_{k}^{3} .
$$

It is enough to show that $0<y_{i} \leq x_{i}$, the vectors $\left(x_{i}, y_{i}\right)$ are different and $\left(64^{k-1}+1\right) w_{k}^{3}<$ $100^{100^{k}}$. Obviously

$$
\frac{x_{i+1}}{y_{i+1}}=\frac{u_{i+1}}{v_{i+1}}=\frac{u_{i}}{v_{i}} \cdot \frac{u_{i}^{3}+2 v_{i}^{3}}{2 u_{i}^{3}+v_{i}^{3}} \cdot \frac{u_{i}^{3}\left(u_{i}^{3}+2 v_{i}^{3}\right)^{3}-2 v_{i}^{3}\left(2 u_{i}^{3}+v_{i}^{3}\right)^{3}}{2 u_{i}^{3}\left(u_{i}^{3}+2 v_{i}^{3}\right)^{3}-v_{i}^{3}\left(2 u_{i}^{3}+v_{i}^{3}\right)^{3}} .
$$

We will show by induction that $u_{i} \geq 4^{k-i} v_{i}$ (and therefore $u_{i} \geq v_{i}$ ) and $u_{i}, v_{i}>0$. This is trivial for $i=1$. Let suppose that $u_{i} \geq 4^{k-i} v_{i} \geq 4 v_{i}$ for some $1 \leq i \leq k$. Then we have

$$
u_{i}^{3}\left(u_{i}^{3}+2 v_{i}^{3}\right)^{3}-2 v_{i}^{3}\left(2 u_{i}^{3}+v_{i}^{3}\right)^{3}>0
$$

and therefore by (10) we have

$$
\frac{u_{i+1}}{v_{i+1}} \geq \frac{u_{i}}{v_{i}} \cdot \frac{1}{2} \cdot \frac{1}{2}
$$

which shows the inductive step. On the other hand in view of (10) we can see that $\frac{u_{i+1}}{v_{i+1}}<\frac{u_{i}}{v_{i}}$, and therefore we have $k$ different vectors $\left(x_{i}, y_{i}\right)$. To finish the proof we have to verify $\left(64^{k-1}+1\right) w_{k}^{3}<100^{100^{k}}$. We know $w_{i+1} \leq 54 u_{i}^{15} w_{i}$ and therefore

$$
w_{k} \leq 54^{k-1} u_{k-1}^{15} u_{k-2}^{15} \ldots u_{1}^{15} w_{1} \leq 54^{k-1} u_{k-1}^{15(k-1)}
$$

Since $u_{i+1} \leq 81 u_{i}^{16}$, we get

$$
u_{k} \leq 81^{1+16+16^{2}+\cdots+16^{k-2}} \leq 81^{\frac{16^{k-1}}{15}}
$$

thus

$$
\left(64^{k-1}+1\right) w_{k} \leq\left(64^{k-1}+1\right) 54^{k-1} 81^{(k-1) 16^{k-1}} \leq 100^{100^{k}},
$$

which completes the proof.

Proof of Theorem 9. To prove Theorem 9 we use the probabilistic method due to Erdős and Rényi. The method is standard therefore we does not give the probabilistic background here (see e.g. the excellent book of Halberstam and Roth [17]). We denote 
the probability of an event by $\mathbb{P}$, and the expectation of a random variable $\zeta$ by $\mathbb{E}(\zeta)$. Let $\Omega$ denote the set of the strictly increasing sequences of positive integers. Theorem 13. in [17], p. 142 . shows that one can obtain a valid probability space $(\Omega, X, \mathbb{P})$, where the events $\mathcal{E}^{(n)}=\{\mathcal{A}: \mathcal{A} \in \Omega, n \in \mathcal{A}\}$ are independent for $n=1,2, \ldots$ We denote the characteristic function of the event $\mathcal{E}^{(n)}$ by $\varrho(\mathcal{A}, n)$ :

$$
\varrho(\mathcal{A}, n)=\left\{\begin{array}{c}
1, \text { if } n \in \mathcal{A} \\
0, \text { if } n \notin \mathcal{A} .
\end{array}\right.
$$

Furthermore, for some $\mathcal{A}=\left\{a_{1}, a_{2}, \ldots\right\} \in \Omega$ denote by $A(n)$ the number of elements of $\mathcal{A}$ up to $n$, i.e.,

$$
A(n)=\sum_{\substack{a \in \mathcal{A} \\ a \leq n}} 1
$$

It is clear that

$$
A(n)=\sum_{j=1}^{n} \varrho(\mathcal{A}, j)
$$

is the sum of Boolean random variables. We need two basic results of probability theory.

Lemma 3. (Borel-Cantelli) Let $X_{1}, X_{2}, \ldots$ be a sequence of events in a probability space. If

$$
\sum_{j=1}^{+\infty} \mathbb{P}\left(X_{j}\right)<\infty
$$

then with probability 1 , at most a finite number of the events $X_{j}$ can occur.

See [17], p. 135. The next tool is the well-known correlation inequality of Chernoff.

Lemma 4. (Chernoff's inequality) If $t_{i}$ 's are independent Boolean random variables and $X=t_{1}+\ldots+t_{n}$, then for any $\delta>0$ we have

$$
\mathbb{P}(|X-\mathbb{E}(X)| \geq \delta \mathbb{E}(X)) \leq 2 e^{-\min \left(\delta^{2} / 4, \delta / 2\right) \mathbb{E}(X)} .
$$

See in [1]. Define the random sequence $\mathcal{A}$ by $\mathbb{P}(\{\mathcal{A}: \mathcal{A} \in \Omega, n \in \mathcal{A}\})=\mathbb{P}(n \in \mathcal{A})=\frac{1}{3} \frac{1}{n^{2 / 3}}$ for every positive integer $n$. It is easy to see that

$$
\mathbb{E}(A(x))=\sum_{n=1}^{x} \frac{1}{3} \frac{1}{n^{2 / 3}}=\int_{1}^{x} \frac{1}{3} y^{-2 / 3} d y+O(1)=x^{1 / 3}+O(1) .
$$

As $A(x)$ is the sum of independent Boolean random variables, it follows from Chernoff's inequality with $\delta=\frac{3}{x^{1 / 6}} \sqrt{\log x}$, that

$$
\begin{gathered}
\mathbb{P}\left(|A(x)-\mathbb{E}(A(x))| \geq \frac{3}{x^{1 / 6}} \sqrt{\log x} \mathbb{E}(A(x))\right) \leq 2 e^{-\frac{9}{4} x^{1 / 6} \log x \mathbb{E}(A(x))} \\
<2 e^{-\frac{9}{4} x^{-1 / 3}(\log x)\left(x^{1 / 3}+O(1)\right)}<e^{-2 \log x} \leq \frac{1}{x^{2}}
\end{gathered}
$$


if $x$ is large enough. By the Borel - Cantelli lemma we have

$$
A(x)=\mathbb{E}(A(x))+O\left(x^{1 / 6} \sqrt{\log x}\right) .
$$

with probability 1 for every $x \geq 2$. It is clear that

$$
n=A\left(a_{n}\right)=a_{n}{ }^{1 / 3}+O\left(a_{n}^{1 / 6} \sqrt{\log a_{n}}\right) .
$$

It follows that

$$
a_{n}^{1 / 3}=n+O(\sqrt{n \log n})
$$

thus we have

$$
a_{n}=n^{3}+O\left(n^{5 / 2} \sqrt{\log n}\right) .
$$

On the other hand we put

$$
r_{2}(\mathcal{A}, n)=\sum_{1 \leq j<n / 2} \varrho(\mathcal{A}, j) \varrho(\mathcal{A}, n-j),
$$

which is also a random variable. It is easy to see that

$\mathbb{E}\left(r_{2}(\mathcal{A}, n)\right)=\frac{1}{9} \sum_{1 \leq j<n / 2} \frac{1}{j^{2 / 3}(n-j)^{2 / 3}} \ll \frac{1}{n^{2 / 3}} \sum_{1 \leq j<n / 2} \frac{1}{j^{2 / 3}}=\frac{1}{n^{2 / 3}}\left(\int_{1}^{n / 2} j^{-2 / 3} d j+O(1)\right) \ll n^{-1 / 3}$.

Let $E_{i}$ be the event

$$
E_{i}=\{i \in \mathcal{A}, n-i \in \mathcal{A}\}
$$

It is clear that the events $E_{i}$ 's are mutually independent. Thus we have

$$
\begin{aligned}
\mathbb{P}\left(r_{2}(\mathcal{A}, n)>3\right) & \leq \sum_{1 \leq i_{1}<i_{2}<i_{3}<i_{4}<n / 2} \mathbb{P}\left(E_{i_{1}} \cap \ldots \cap E_{i_{4}}\right)=\sum_{1 \leq i_{1}<i_{2}<i_{3}<i_{4}<n / 2} \mathbb{P}\left(E_{i_{1}}\right) \ldots \mathbb{P}\left(E_{i_{4}}\right) \\
& \leq\left(\frac{1}{9} \sum_{1 \leq j<n / 2} \frac{1}{j^{2 / 3}(n-j)^{2 / 3}}\right)^{4}=\mathbb{E}\left(r_{2}(\mathcal{A}, n)\right)^{4} \ll n^{-4 / 3} .
\end{aligned}
$$

It follows from the Borel - Cantelli lemma that with probability 1 , for $n>n_{0}, r_{2}(\mathcal{A}, n) \leq$ 3. This implies that $R_{\mathcal{A}}(n)$ is also bounded and so does $s_{\mathcal{A}}(n)$.

\section{References}

[1] N. Alon - J. Spencer, The Probabilistic method, Wiley Interscience (2000).

[2] P. Borwein, S. Choi, F. Chu, An old conjecture of Erdös - Turán on additive bases, Math. Comp. 75 (2006), 475-484.

[3] Y-G. Chen, The analogue of Erdös - Turán conjecture in $\mathbb{Z}_{m}$, J. Number Theory, 128 (2008), 2573-2581.

[4] Y-G. Chen, On the Erdös - Turán conjecture, C. R. Math. Acad. Sci. Paris 350 (2012), 933-935. 
[5] G. A. Dirac, Note on a problem in additive number theory, J. London Math. Soc., 26 (1951), 312-313.

[6] M. Dowd, Questions related to the Erdös - Turán conjecture, SIAM J. Discrete Math., 1 (1988), 142-150.

[7] P. Erdős, P. Turán, On a problem of Sidon in additive number theory, and some related problems, J. London Math. Soc. 16 (1941), 212-215.

[8] P. Erdős, Problems and results in additive number theory, in: Colloque sur la Théorie des Nombres, Bruxelles, 1955, George Thone, Liége; Masson and Cie, Paris, 1956, pp. $127-137$.

[9] P. Erdős, W. H. J. Fuchs, On a problem of additive number theory, J. London Math. Soc., 31 (1956), 67-73.

[10] G. Grekos, L. Haddad, C. Helou, J. Pihko, On the general Erdös - Turán conjecture, International Journal of Combinatorics, (2014) Art. ID 826141, 11 pp.

[11] G. Grekos, L. Haddad, C. Helou, J. Pihko, On the Erdős - Turán conjecture, J. Number Theory 102 (2003), 339-352.

[12] G. Grekos, L. Haddad, C. Helou, J. Pihko, The class of Erdös - Turán sets, Acta Arith., 117 (2005), 81-105.

[13] G. Grekos, L. Haddad, C. Helou, J. Pihko, Variations on a theme of Cassels for additive bases, Intl. J. Number Theory 2 (2006), 249-265.

[14] L. Haddad, C. Helou, Bases in some additive groups and the Erdős - Turán conjecture, J. Combin. Theory Ser. A 108 (2003), 339-352.

[15] L. Haddad, C. Helou, Additive bases representations in groups, Integers 8 (2008), A5.

[16] L. Haddad, C. Helou, Representations of integers by near quadratic sequences, Journal of Integer sequences $\mathbf{1 5}$ (2012), 12.8.8.

[17] H. Halberstam, K. F. Roth, Sequences, Springer - Verlag, New York, 1983.

[18] S. F. Konyagin and V. F. Lev, The Erdős - Turán problem in infinite groups, in: Additive Number Theory, Springer 20 (2010), 195 - 202.

[19] M. B. Nathanson, Unique representation bases for the integers, Acta Arith., 108 (2003), $1-8$.

[20] M. B. Nathanson, Representation functions of additive bases for abelian semigroups, Int. J. Math. Sci. (2004), 1589 - 1597.

[21] M. B. Nathanson, Generalized additive bases, König's lemma and the Erdös - Turán conjecture, 106 (2004), 70 - 78. 
[22] J.Nesetril and O. Serra, On a conjecture of Erdős and Turán for additive basis, in Proceedings of the "Segundas Jornadas de Theoria de Números", Bibl. Rev. Mat. Iberoamericana, (2008), 209 - 220.

[23] I. Niven, H. S. Zuckerman, H. L. Montgomery, An Introduction to The Theory of Numbers, 5th ed., Wiley, 1991.

[24] V. Pus, On multiplicative bases in abelian groups, Czech. Math. J. 41 (1991), 282 287.

[25] Cs. Sándor, A note on a conjecture of Erdös and Turán, Integers, 8 (2008), A30.

[26] T. Min, On the Erdős - Turán conjecture, J. of Number Theory, 150 (2015), 74 - 80.

[27] G. Tenenbaum, Introduction to analytic and probabilistic number theory, Cambridge University Press; 1st ed. 1995.

[28] Y. Quan-Hui, A generalization of Chen's theorem on the Erdös-Turán conjecture, Int. J. Number Theory 9 (2013), 1683 - 1686. 